\title{
Sexual activity among patients in psychiatric hospital wards
}

\author{
James Warner MD MRCPsych ${ }^{1}$ \\ Nicola Pitts BSc MBBS ${ }^{1} \quad$ Mike J Crawford MD MRCPsych ${ }^{1}$ \\ Marc Serfaty MPhil MRCPsych² \\ Pramod Prabhakaran MB MRCPsych ${ }^{3}$ Rizkar Amin MB MRCPsych ${ }^{3}$
}

J R Soc Med 2004;97:477-479

\section{SUMMARY}

In psychiatric hospitals, sexual activity between patients raises special difficulties regarding consent. We undertook a questionnaire survey of inpatients in the mental health units of three hospitals to identify the nature and frequency of sexual activity. A contemporaneous staff questionnaire was used in an attempt to validate the patient reports.

Of the 100 patients who participated (response rate 60\%), 30 reported engaging in some form of sexual activity including 10 who had sexual intercourse. All sexual intercourse was consensual, but only 2 respondents used condoms. Staff questionnaires suggested levels of sexual activity congruent with patient reports.

This survey underlines the conflict between an individual's right to sexual expression and the need to protect vulnerable patients.

\section{INTRODUCTION}

There is a dearth of evidence on sexual activity among patients on psychiatric units although staff-based reports have suggested it may be quite common. ${ }^{1-5}$ Although article 8.1 of the European Convention on Human Rights emphasizes the individual's 'right to respect for the private life', widely interpreted as a right to sexual expression among consenting adults, the milieu of psychiatric wards is likely to prove more restrictive. ${ }^{6}$ Healthcare providers have to balance the freedom of sexual expression among adults able to give consent against the risks of sexual exploitation, bartering, sexually transmitted diseases and unwanted conception. Until levels of activity are known, health providers and clinicians will be unable to offer safe environments for patients. We aimed to identify the levels of sexual activity among a representative sample of psychiatric inpatients in three hospitals.

\section{METHODS}

The study was conducted in eleven wards at three psychiatric units across West London. On being approached, all wards agreed to participate, and the investigation had the approval of the participating trusts and

${ }^{1}$ Department of Psychiatry, Imperial College London; ${ }^{2}$ Department of Behavioural Sciences and Psychological Medicine, Royal Free and UCL Medical School, London; ${ }^{3}$ Central and North West London Mental Health Trust, London, UK

Correspondence to: Dr James Warner, Senior Lecturer in Psychiatry, Imperial College London, Paterson Centre, 20 South Wharf Road, London W2 1PD, UK

E-mail j.warner@imperial.ac.uk local research ethics committees. A questionnaire seeking information about sexual activity among patients was developed by a focus group of clinicians. Responses were either dichotomous or made on a four-point Likert scale from $1=$ strongly agree to $4=$ strongly disagree. The questionnaire was piloted on 5 patients to ensure that the language and format were appropriate, but no important changes were required. The questionnaire sought information on: personal background (age, diagnosis, gender, length of hospital stay); whether the respondent had received any sexual advances while in hospital, and, if so, the characteristics of that advance and how it was received; whether the respondent had made any sexual advances while in hospital; and details of any joint sexual activities while in hospital (foreplay, mutual masturbation, oral sex, intercourse) including timing and location, relationship with partner, contraceptive usage and consent to activity. In addition to the above information, researchers $(\mathrm{NP}, \mathrm{JW}, \mathrm{PP})$ recorded information on the ward type (acute adult/acute elderly/secure/rehabilitation) for each respondent.

\section{Staff questionnaire}

A thirteen item questionnaire was designed for completion by ward nursing staff. They were asked whether they thought the psychiatric inpatients engaged in sexual activity and, if so, the nature of the activity and how often. The questionnaire also sought information on staff attitudes to patients having sex in hospital settings, provision of facilities and policing of this activity. 


\section{Samples and data analysis}

Data collection took place over four weeks, during which researchers visited the participating wards on several occasions to maximize recruitment. Individual researchers were matched to specific wards so as to reduce the possibility of patients' completing the survey twice. The patient questionnaire was offered to all individuals who had been inpatients for more than five days and were present on the wards at the time of researchers' visits. Patients thought incapable of providing informed consent for participation in the study $(n=3)$ were excluded. Patients were approached individually, provided with an information sheet and given the opportunity to complete the questionnaire in a private setting. To preserve anonymity, names of respondents were not recorded; with the approval of the research ethics committees, they were asked for verbal rather than written consent. Paper-based and computerized versions of the questionnaire were available and these took 15-20 minutes to complete. To gauge the representativeness of this sample, we compared demographic characteristics with all admissions to the participating units over the previous year. The staff questionnaire was offered to all nursing staff present on the wards at the time of a researcher's visit.

Computerized questionnaire data were entered into EpiData version 2.1 and paper-based questionnaires were put into the same program by a researcher. Numerical data were then exported to SPSS version 10.0 for analysis. We used Pearson chi-squared and Mann-Whitney $U$-tests to analyse non-parametric data and independent sample $t$-tests for normal data.

\section{RESULTS}

Of the 167 patients approached, $100(60 \%)$ agreed to participate - median length of admission 31 days (range 5-360), mean age 39 (SD 14), 61 male. This sample was demographically similar to the admission population over the previous year $-n=554$, mean age 42 (SD 15), $54 \%$ male. Diagnoses given by respondents included schizophrenia $(n=32)$, depression $(n=32)$ and bipolar disorder $(n=18)$. Some respondents declined to provide a diagnosis.

Table 1 gives details of sexual activity. Of the 10 respondents who reported having sexual intercourse, all said this activity was consensual. 8 used no contraception; of the others, two reported using a condom and one oral contraceptives. Men and women were equally likely to report sexual activity. 6 respondents said the sexual intercourse took place within a long-term relationship established on the ward; 3 reported intercourse on more than five occasions. Locations for activity included bedroom (events $n=40)$, dayroom $(n=46)$, toilet $(n=25)$ and outside the immediate ward (e.g. stairwell, garden $n=35$ ). No respondents reported having sex with staff members and no association was found between ward type, length of stay, diagnosis or gender and sexual activity.

Of 27 staff members who completed questionnaires, 26 believed sexual activity to be occurring in the psychiatric unit; 13 thought it happened often. Of those who believed sexual activity occurred on the wards, 16 judged that this included sexual intercourse. All the units surveyed had a 'no sex' policy.

\section{DISCUSSION}

The amount of sexual activity disclosed by the survey was remarkably high. Although all sexual intercourse was rated as consensual by respondents, some sexual activity was nonconsensual. We found no relation between length of stay, diagnosis or ward type and levels of invitation to, or participation in, sexual activity. This may reflect the small sample size, but also indicates that all patients may be vulnerable to sexual exploitation.

Validation of questionnaires of this nature is difficult: some individuals will under-report because of concern about privacy, others may exaggerate. Previous estimates of sexual activity, based on reports by staff alone, have been

Table 1 Reported rates of joint sexual activity among $\mathbf{1 0 0}$ psychiatric inpatients

\begin{tabular}{|c|c|c|c|c|c|}
\hline \multirow[b]{2}{*}{ Activity } & \multicolumn{2}{|c|}{ Invitation from others } & \multirow{2}{*}{$\begin{array}{l}\text { Advances to } \\
\text { others }\end{array}$} & \multicolumn{2}{|c|}{ Actual activity } \\
\hline & Total & Unwanted & & Total & Unwanted \\
\hline All activities & 38 & 6 & 21 & 30 & 6 \\
\hline Kissing & 21 & 2 & 11 & 19 & 6 \\
\hline Other foreplay & 11 & 0 & 7 & 7 & 0 \\
\hline Mutual masturbation & 6 & 0 & 4 & 7 & 1 \\
\hline Oral sex & 9 & 0 & 3 & 9 & 0 \\
\hline Intercourse & 11 & 0 & 5 & 10 & 0 \\
\hline
\end{tabular}


lower. ${ }^{3-5}$ In a retrospective survey by Akhtar et al. ${ }^{3}$ only $3 \%$ of psychiatric patients were believed to have engaged in sexual activity of any sort. In a 2-year prospective survey, again of staff, Keitner et al..$^{5}$ found that about $8 \%$ of patients had engaged in physical relationships. The credibility of our own estimate is strengthened by the concordance between patient and staff responses. It is noteworthy that Akhtar and Keitner conducted their surveys in the 1970s and 1980s. The far higher levels of patient sexual activity identified in our staff survey may reflect greater openness in discussing sexual matters, or greater vigilance among nursing staff, or a change in the behaviour of patients.

This survey is small, and is weakened by the low response rate. The reason $40 \%$ of patients declined to take part may be that they did not regard the study as personally relevant. Furthermore, we were unable to approach a small number of patients who were so severely ill they were unable to give consent to the survey - although this group may be at greater risk of exploitation. A further difficulty arose from the anonymity of respondents. We judged this paramount to the success of the study - not least because the participants might have feared censure or provided incorrect information if they thought responses could be fed back to the clinical teams. Nevertheless, it did mean respondents might in theory have completed the survey more than once, although the study was conducted over a short time-frame and as far as practicable a single researcher interviewed every participant on a particular ward. We were unable to compare clinical or demographic characteristics of respondents and those who declined to participate.
Even if the estimates from the current survey are misleadingly high, because of an unrepresentative sample, the absolute number of patients engaging in sexual activity demands attention. For some of these, especially those unable to give consent or detained under the Mental Health Act 1983, sexual activity may be illegal, and health care providers have an obligation to protect them from sexual exploitation. Furthermore, health care trusts may be held responsible if a patient under their care engages in sexual activity and consequently becomes pregnant or develops a sexually transmitted disease. But for others any attempt at policing might violate the European Convention on Human Rights. Given the fluctuating nature of capacity, providers face a near-impossible task in balancing these issues. We urge hospitals to revise their 'no sex' policies, accept that sex takes place on psychiatric units and ensure that condoms and contraceptive advice are available.

\section{REFERENCES}

1 Buckley PF, Hyde JL. State hospitals' response to the sexual behaviour of psychiatric inpatients. Psychiatric Services 1997;48:398-9

2 Civic D, Walsh G, McBride D. Staff perspectives on sexual behaviour of patients in a state psychiatric hospital. Hosp Commun Psychiatry 1993;44:887-9

3 Akhtar S, Crocker E, Dickey N, Helfrich J, Rheuban W. Overt sexual behaviour among psychiatric inpatients. Dis Nerv Syst 1977;38:359-61

4 Modestin J. Patterns of overt sexual interaction among acute psychiatric inpatients. Acta Psychiatr Scand 1981;64:446-59

5 Keitner GI, Baldwin LM, McKendall MJ. Copatient relationships on a short-term psychiatric unit. Hosp Commun Psychiatry 1986;37:166-70 